Document downloaded from:

http://hdl.handle.net/10251/50931

This paper must be cited as:

Roger Varea, S.; Calabuig Soler, D.; Cabrejas Peñuelas, J.; Monserrat Del Río, JF. (2014). Multi-user non-coherent detection for downlink MIMO communication. IEEE Signal Processing Letters. 21(10):1225-1229. doi:10.1109/LSP.2014.2330854.

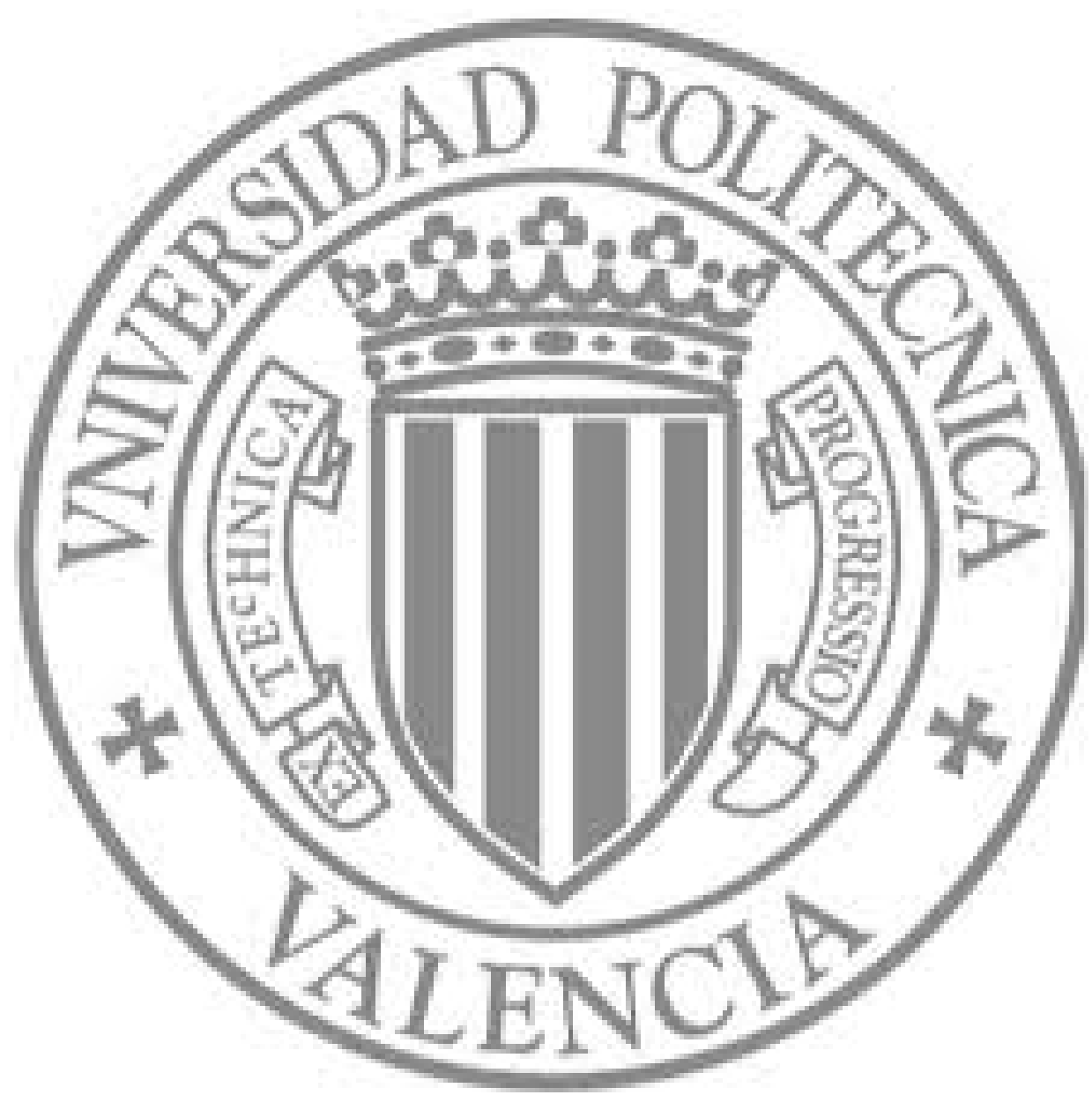

The final publication is available at

http://dx.doi.org/10.1109/LSP.2014.2330854

Copyright Institute of Electrical and Electronics Engineers (IEEE) 


\title{
Multi-user Non-coherent Detection for Downlink MIMO Communication
}

\author{
Sandra Roger, Daniel Calabuig, Member, IEEE, Jorge Cabrejas, and Jose F. Monserrat, Senior \\ Member, IEEE
}

\begin{abstract}
Current cellular technologies are based on the concept of coherent communication, in which the channel matrix used for demodulation is estimated via reference or pilot signals. Coherent systems, however, involve a significant increase of the signalling overhead, especially when the number of transmission points is increased or when the mobile channel changes rapidly, which motivates the use of non-coherent techniques. This letter extends the use of non-coherent communications to a multi-user (MU) multiple-input multiple-output (MIMO) framework by combining superposition coding with a reduced-complexity detection method. Numerical results confirm that our scheme achieves higher user rates than non-coherent MU transmission based on time multiplexing. In addition to the well-known sumrate gain of MU systems, an extra performance gain given by downlink non-coherent MU communication is shown and qualitatively justified.
\end{abstract}

\section{Index Terms}

Non-coherent MIMO communication, multi-user detection, space-time coding.

\section{INTRODUCTION}

The estimation of channel coefficients for coherent communication is usually performed by including into the data stream some reference or pilot symbols already known by the receiver. Although pilot-based channel estimation has been broadly adopted by current wireless systems, its application to multiple-input multiple-output (MIMO) systems entails some challenges and practical limitations [1]. In fact, channel estimation complexity increases with the number of transmit and receive antennas. Moreover, channel estimation errors can degrade the performance of MIMO techniques substantially. Therefore, coherent techniques may prove impractical in fast fading scenarios and, thus, transmission techniques dependent on the channel coefficients knowledge are usually discarded for vehicular communications [2]. All these drawbacks have prompted an increasing interest towards the research

(C)2014 IEEE. Personal use of this material is permitted. However, permission to use this material for any other purposes must be obtained from the IEEE by sending a request to pubs-permissions@ieee.org.

The authors are with the Institute of Telecommunications and Multimedia Applications, Universitat Politècnica de València, Valencia, 46022 Spain, e-mails: $\{$ sanrova, dacaso, jorcabpe, jomondel\}@iteam.upv.es.

This work was performed in the framework of the FP7 project ICT-317669 METIS, which is partly funded by the European Union. 
on non-coherent communication, which performs data detection without any knowledge of the channel coefficients. In fact, various forums already identified non-coherent techniques as a potential enabler for the future 5th generation mobile networks (5G) [3], [4], where non-coherent schemes may be a good complement to coherent communication in some scenarios.

Pioneering works on non-coherent communication techniques showed that a proper design of the transmitted signals allows to approach the capacity of coherent systems at high signal-to-noise ratios (SNR) [5]. Among the many constellation designs for non-coherent MIMO communication, differential unitary space-time modulation (DUSTM) is of particular interest [6]. The main advantage of DUSTM is its efficient decoding, which can be carried out through multiple-symbol differential detection (MSSD) at the receiver side [7]. Other meaningful schemes for non-coherent MIMO communication are based on Grassmannian Constellations (GC), which can be designed in either a systematic [8], [9] or a non-systematic way [10]. Regarding the decoding of GC, these designs cannot exploit MSSD due to their non-differential construction. Nevertheless, GC can be non-coherently decoded using generalized likelihood ratio test (GLRT) receivers. To the best of the authors' knowledge, previously proposed GLRT-based GC detection techniques are all intended for single-user (SU) non-coherent communication.

In current cellular systems, multi-user (MU) communication increases the overall system performance with respect to its SU counterpart. However, the extension of previously proposed SU non-coherent MIMO constellations and detection techniques to allow MU operation is not straightforward and, thus, they need to be redefined. This paper proposes the use of superposition coding [11] to allow the application of SU constellations in a MU setup. Furthermore, this paper also presents the maximum likelihood (ML) detection rule for MU communication in a non-coherent framework. This rule is shown to have an exponential complexity with respect to the number of users, hence, its evaluation is unfeasible for a relatively small number of users. For that reason, a suboptimal but feasible detector is proposed, which reduces complexity from exponential to linear. Finally, this paper studies the MU gain, which is shown to have an extra factor in the case of downlink non-coherent communications. This factor is a consequence of the channel information that can be extracted from the transmissions to other users. Numerical results show that this information can be used to reduce the block error rate (BLER) in comparison with the SU case.

\section{SySTEM MODEL}

We consider a MIMO downlink transmission from a base station (BS) with $M$ antennas to $K$ user equipment (UE) devices with $N$ antennas each. We assume a transmission through block-fading channels $\mathbf{H}_{k} \in \mathbb{C}^{M \times N}$, $k=1, \ldots, K$, containing Gaussian i.i.d. elements with zero mean and unit variance, which are unknown at both the transmitter and receiver sides. The channel block length is assumed to be $T$ consecutive channel uses. The signal transmitted to user $k$ through the $M$ antennas during one channel block is represented by the matrix $\mathbf{X}_{k} \in \mathbb{C}^{T \times M}$. The possible transmitted matrices are uniformly drawn from a finite-length constellation $\mathcal{C}=\left\{\mathbf{S}_{i}, i=1, \ldots,|\mathcal{C}|\right\} \subset$ $\mathbb{C}^{T \times M}$, the elements of which have covariance $\Gamma_{\mathrm{X}}$ and, for simplicity, zero mean. For further ease of exposition, in this letter we assume all users' signals are drawn from the same constellation. Nevertheless, the results presented 
in this paper could be easily extended to other constellations with non-zero mean elements and, also, to different per-user constellations.

One of the main objectives of this paper is to provide MU communication with non-coherent reception. Following the rationale of [11], in the Gaussian broadcast channel with multiple receivers, superposing messages with different power levels generally improves the achievable rates in comparison with time sharing procedures, provided that successive decoding at the receivers is feasible [12]. With the aim of validating this conclusion under non-coherent detection, in this work we combine superposition coding [11] with GC, i.e. the transmitted signal is a superposition of independent GC signals. To this end, the BS power has to be divided among the users. We define different per-user power sharing factors $\gamma_{k}, k=1, \ldots, K$, so that $\sum_{k=1}^{K} \gamma_{k}=1$. We further consider an SNR equal to $\rho_{k}$ at user $k$, where the power contributions of all the users are included within the desired signal power term. User $k$ is assumed to know $\rho_{k}, \gamma_{\ell}$ for $\ell=1, \ldots, K$ and also the constellation.

The received signal matrix for user $k, \mathbf{Y}_{k} \in \mathbb{C}^{T \times N}$, is

$$
\mathbf{Y}_{k}=\left(\sum_{\ell=1}^{K} \sqrt{\frac{\gamma_{\ell} \rho_{k} T}{\operatorname{Tr}\left(\boldsymbol{\Gamma}_{\mathbf{X}}\right)}} \mathbf{X}_{\ell}\right) \mathbf{H}_{k}+\mathbf{Z}_{k}
$$

where $\mathbf{Z}_{k} \in \mathbb{C}^{T \times N}$ is the additive white Gaussian noise (AWGN) matrix with i.i.d. zero-mean unit-variance elements.

\section{Non-COHEREnt MUlti-USER Receiver}

In this section, we first address the non-coherent ML detection rule for the investigated MU scheme. A quantitative analysis of its computational cost is also provided. Then, a suboptimal detection scheme suitable for the considered superposition coding setup is proposed and shown to reduce the ML detector computational cost.

\section{A. Maximum-Likelihood Non-coherent Multi-user Detection}

Focusing on the data detection at a certain user $k$, the symbol detected by the ML receiver maximizes the following conditioned probability density function (pdf):

$$
\hat{\imath}_{k}=\arg \max _{i} f_{\mathbf{Y}_{k}}\left(\mathbf{\Upsilon} \mid \mathbf{X}_{k}=\mathbf{S}_{i}\right),
$$

where $\Upsilon$ denotes a particular value taken by the random variable $\mathbf{Y}_{k}$. For the sake of simplicity, we introduce $\mathbf{X}=$ $\left(\mathbf{X}_{1}, \ldots, \mathbf{X}_{K}\right) \in \mathcal{C}^{K}$, which contains all the users' signals. We further introduce $\mathbf{X}_{-k}=\left(\mathbf{X}_{1}, \ldots, \mathbf{X}_{k-1}, \mathbf{X}_{k+1}, \ldots, \mathbf{X}_{K}\right) \in$ $\mathcal{C}^{K-1}$. Since $\mathcal{C}$ is a finite-length constellation with equiprobable symbols, so are $\mathcal{C}^{K}$ and $\mathcal{C}^{K-1}$.

Taking into account these considerations, $f_{\mathbf{Y}_{k}}\left(\mathbf{\Upsilon} \mid \mathbf{X}_{k}=\mathbf{S}_{i}\right)$ can be obtained as follows:

$$
f_{\mathbf{Y}_{k}}\left(\mathbf{\Upsilon} \mid \mathbf{X}_{k}=\mathbf{S}_{i}\right)=\frac{1}{\left|\mathcal{C}^{K-1}\right|} \sum_{\mathbf{A} \in \mathcal{C}^{K-1}} f_{\mathbf{Y}_{k}}\left(\mathbf{\Upsilon} \mid \mathbf{X}_{-k}=\mathbf{A}, \mathbf{X}_{k}=\mathbf{S}_{i}\right)
$$

For the Gaussian random channel, the conditioned probability terms in (3) follow a Gaussian distribution [5], that is,

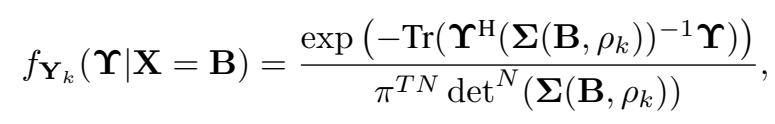


where, $\boldsymbol{\Sigma}\left(\mathbf{B}, \rho_{k}\right)=E\left[\mathbf{Y}_{k} \mathbf{Y}_{k}^{\mathrm{H}} \mid \mathbf{X}=\mathbf{B}\right]$ denotes the conditioned covariance matrix of the received signal $\mathbf{Y}_{k}$. The term $\boldsymbol{\Sigma}\left(\mathbf{B}, \rho_{k}\right)$ can be directly obtained from

$$
\boldsymbol{\Sigma}(\mathbf{B}, \rho)=\frac{N \rho T}{\operatorname{Tr}\left(\boldsymbol{\Gamma}_{\mathrm{X}}\right)} \sum_{m=1}^{K} \sum_{n=1}^{K} \sqrt{\gamma_{m} \gamma_{n}} \mathbf{B}_{m} \mathbf{B}_{n}^{\mathrm{H}}+N \mathbf{I} .
$$

Here, the notation $\mathbf{B}_{j}$ has been used to reference the $j$-th $T \times M$-dimensional block of $\mathbf{B}$ and $\mathbf{I}$ denotes the $T \times T$ identity matrix.

Let us now focus on the computational burden of the derived non-coherent MU ML detector. It can be observed that the ML detection of each user's signal in this MU framework involves the computation of (3) a number of times equal to $|\mathcal{C}|$, i.e. one per constellation symbol. Hence, this detection technique leads to an overall per user cost of $|\mathcal{C}|^{K}$ evaluations of the function (4) and, implicitly, also of (5). Note that such a high computational cost, which increases exponentially with the number of users, is impractical for real-world communication systems. In addition, the constellation size has also a big impact on the detection complexity, which may prevent practical systems from transmitting at high rates due to this burdensome detection stage.

\section{B. Proposed Non-coherent Multi-user Detection}

In order to reduce the ML detection complexity, we propose a suboptimal MU receiver adapted to the superposition coding transmission scheme. The key feature of this receiver is that each user detects its own signal after previous detection of the signals of other users, which are received with higher power than its own. To perform this successive detection, an approximated system model is considered at each detection step. It is important to note that, due to the fact that the channel coefficients are unknown, conventional successive interference cancelation is not possible at each detection stage to remove the effect of previously detected user signals. Rather, previously detected symbols are considered within the detection rule. The approximated system model and proposed detection scheme are next described.

Without loss of generality, $\gamma_{1}>\ldots>\gamma_{K}$ is assumed, which means that user $k$ receives the signals for users $1, \ldots, k-1$ with higher power than its own signal, and the signals for users $k+1, \ldots, K$ with lower power. In the proposed detection scheme, user $k$ detects consecutively, and prior to its own signal, the signals of users $p=1, \ldots, k-1$, which are received with higher power than its own. For the detection of user $p$, the signals for users $p+1, \ldots, K$ are considered as noise and, hence, are grouped within the AWGN term in the system model. Hence, user $k$ will consider the following approximated expression of the received signal for the detection of the signal for user $p$ :

$$
\tilde{\mathbf{Y}}_{k, p}=\left(\sum_{\ell=1}^{p} \sqrt{\frac{\gamma_{\ell} \rho_{k} T}{\operatorname{Tr}\left(\boldsymbol{\Gamma}_{\mathbf{X}}\right)\left(1+\sum_{\ell=p+1}^{K} \gamma_{\ell} \rho_{k}\right)}} \mathbf{X}_{\ell}\right) \mathbf{H}_{k}+\mathbf{Z}_{k},
$$

where the power levels of users $p+1, \ldots, K$ reduce the original SNR.

Considering the approximated system model (6) and introducing the function

$$
G(\boldsymbol{\Upsilon}, \mathbf{B}, \rho)=\frac{\exp \left(-\operatorname{Tr}\left(\Upsilon^{\mathrm{H}}(\boldsymbol{\Sigma}(\mathbf{B}, \rho))^{-1} \mathbf{\Upsilon}\right)\right)}{\pi^{T N} \operatorname{det}^{N}(\boldsymbol{\Sigma}(\mathbf{B}, \rho))},
$$


the proposed detection rule to calculate the symbol for user $p$ by user $k$ is

$$
\hat{\imath}_{k, p}=\arg \max _{i} G\left(\mathbf{\Upsilon},\left(\mathbf{S}_{\hat{\imath}_{k, 1}}, \ldots, \mathbf{S}_{\hat{\imath}_{k, p-1}}, \mathbf{S}_{i}, \mathbf{0}, \ldots, \mathbf{0}\right), \rho_{k}^{\prime}\right),
$$

where $\mathbf{0}$ stands for a matrix containing $T \times M$ zero elements and $\rho_{k}^{\prime}=\rho_{k} /\left(1+\sum_{\ell=p+1}^{K} \gamma_{\ell} \rho_{k}\right)$. Recall that $\boldsymbol{\Sigma}(\mathbf{B}, \rho)$ denotes the conditioned covariance matrix described in (5).

Overall, (8) is an approximation of (2) that, instead of summing all the probability terms (4) for every possible constellation values for every user, it takes into account only the particular values of $\hat{\imath}_{k, 1}, \ldots, \hat{\imath}_{k, p-1}$, which are already known due to previous detection steps at user $k$. Note that, at every user, the detection of the signal of user 1 simply solves a SU detection problem, since the rest of users are considered within the noise term. Therefore, the covariance matrix in (5) is reduced to

$$
\boldsymbol{\Sigma}\left(\left(\mathbf{S}_{i}, \mathbf{0}, \ldots, \mathbf{0}\right), \rho_{k}^{\prime}\right)=\frac{N \rho_{k}^{\prime} \gamma_{k} T}{\operatorname{Tr}\left(\mathbf{\Gamma}_{\mathbf{X}}\right)} \mathbf{S}_{i} \mathbf{S}_{i}^{\mathrm{H}}+N \mathbf{I}
$$

After substituting (9) into (7) and assuming unitary codebooks, the maximization problem in (8) turns into the following equivalent detection rule for user 1 :

$$
\hat{\imath}_{k, 1}=\arg \max _{i} \operatorname{Tr}\left(\mathbf{\Upsilon}^{\mathrm{H}} \mathbf{S}_{i} \mathbf{S}_{i}^{\mathrm{H}} \mathbf{\Upsilon}\right),
$$

which corresponds to the SU non-coherent ML receiver considered in [9] and [10]. Since unitary codebooks are assumed in this work, the ML receiver is equivalent to the well-known generalized likelihood ratio test (GLRT) receiver [9].

Remark: If the users transmit different constellations, in the MU ML detector, each user must know the constellations of the $K$ users. In the proposed suboptimum detector, however, user $k$ needs only to know the constellations of users $p=1, \ldots, k-1$ for their previous detection.

Regarding computational aspects, we next evaluate the detection complexity of the worst-case user, i.e. user $K$, which must detect $K-1$ signals prior to its own. In this case, the proposed detector performs $K|\mathcal{C}|$ evaluations of the function $G(\mathbf{\Upsilon}, \mathbf{B}, \rho)$, that is, $|\mathcal{C}|$ function evaluations for each detected user. Therefore, even for the worstcase user, the per-user cost is reduced from exponential to linear with the number of users. Furthermore, note that for users $1, \ldots, K-1$, the calculation of the covariance matrix is far less computationally demanding than in the initial non-coherent MU ML detector, due to all the components of $\mathbf{B}$ that are set to zero. As a result, the proposed MU detector is a meaningful scheme to provide non-coherent detection with much fewer complexity than the exhaustive-search-based ML detection scheme.

\section{Downlink Non-Coherent Multi-User Gain}

In this section, we highlight a special feature of downlink non-coherent MU communications based on superposition coding. We will study this feature using a simplified two-user system model. Let

$$
\mathbf{Y}=\mathbf{W}+\mathbf{X}_{2} \mathbf{H}
$$


be the received signal, where

$$
\mathbf{W}=\mathbf{X}_{1} \mathbf{H}+\mathbf{Z}
$$

$\mathbf{X}_{1}$ and $\mathbf{X}_{2}$ are the independent signals transmitted to the two users, $\mathbf{H}$ is the channel matrix, and $\mathbf{Z}$ is the AWGN. Let $\mathbf{X}_{1}$ be the desired signal to be detected from the received signal $\mathbf{Y}$. Note that $\mathbf{W}$ corresponds to the received signal in a case where only $\mathbf{X}_{1}$ is present in the system ( $\mathrm{SU}$ case) and $\mathbf{Y}$ can be seen as a postprocessed version of $\mathbf{W}$, due to the addition of $\mathbf{X}_{2} \mathbf{H}$.

We first want to prove that, in a coherent system, the signals of the rest of users can never enhance the detection performance of the desired user signal. In other words, other signals in the system are only seen as an additional interference. As observed from (11) and (12), if $\mathbf{H}$ and $\mathbf{W}$ are known, knowing $\mathbf{X}_{1}$ does not provide any extra information about $\mathbf{Y}$, i.e. $\mathbf{X}_{1}$ and $\mathbf{Y}$ are conditionally independent and $f_{\mathbf{Y}}\left(\mathbf{Y} \mid \mathbf{W}, \mathbf{X}_{1}, \mathbf{H}\right)=f_{\mathbf{Y}}(\mathbf{Y} \mid \mathbf{W}, \mathbf{H})$. Therefore, the random variables $\mathbf{X}_{1}, \mathbf{W}$ and $\mathbf{Y}$ form the Markov chain $\mathbf{X}_{1} \rightarrow \mathbf{W} \rightarrow \mathbf{Y}$. As in any other Markov chain, the data processing theorem must be fulfilled, which states that processing $\mathbf{W}$ can not add new information about $\mathbf{X}_{1}$, i.e. $I\left(\mathbf{X}_{1} ; \mathbf{W} \mid \mathbf{H}\right) \geq I\left(\mathbf{X}_{1} ; \mathbf{Y} \mid \mathbf{H}\right)$. As a consequence, superposing $\mathbf{X}_{2} \mathbf{H}$ over $\mathbf{W}$ (11) results in no more capacity in comparison with the case where only $\mathbf{X}_{1}$ is transmitted (12). Hence, the capacity of user 1 in the MU coherent setup is never higher than in the SU coherent setup. A similar conclusion can be drawn for uplink MU communications, in either the coherent or non-coherent case.

Now we focus on the downlink non-coherent case. If $\mathbf{W}$ is known but $\mathbf{H}$ is unknown, $\mathbf{Y}$ still has dependence on $\mathbf{X}_{1}$ through $\mathbf{H}$. In this non-coherent case, the knowledge about $\mathbf{X}_{1}$ and $\mathbf{W}$ can provide some extra information about the channel matrix $\mathbf{H}$, or, equivalently, $f_{\mathbf{Y}}\left(\mathbf{Y} \mid \mathbf{W}, \mathbf{X}_{1}\right) \neq f_{\mathbf{Y}}(\mathbf{Y} \mid \mathbf{W})$. Therefore, the variables $\mathbf{X}_{1}, \mathbf{W}$ and $\mathbf{Y}$ do no longer form a Markov chain, as opposed to what happens in the coherent scenario. Consequently, the data processing inequality does not necessarily hold and both conditions $I\left(\mathbf{X}_{1} ; \mathbf{W}\right) \geq I\left(\mathbf{X}_{1} ; \mathbf{Y}\right)$ and $I\left(\mathbf{X}_{1} ; \mathbf{W}\right)<I\left(\mathbf{X}_{1} ; \mathbf{Y}\right)$ are attainable. This means that, in the downlink MU non-coherent setup, it is also possible to increase the information about $\mathbf{X}_{1}$ by processing $\mathbf{W}$. Note that a more elaborated study on the situations where $I\left(\mathbf{X}_{1} ; \mathbf{W}\right)<I\left(\mathbf{X}_{1} ; \mathbf{Y}\right)$ is out of the scope of this letter. Nevertheless, in the following section we will show through simulations an exemplary case where the BLER of an individual user in a two-user setup is reduced with respect to the SU case. This happens when there is a high difference between the power of the two users; the signal $\mathbf{X}_{2}$ increases the channel knowledge for the detection of $\mathbf{X}_{1}$.

\section{RESULTS}

In this section some numerical results that show the performance of the proposed MU detection schemes are presented. A block-fading Gaussian channel constant during $T=4$ time slots is considered to evaluate the transmission from $M=2$ antennas to $K=2$ users with $N=2$ antennas each. The selected codebook for both users is a GC of 16 points, obtained using the direct design proposed in [10]. Note that the use of this codebook achieves a maximum transmission rate of $R_{\mathrm{c}}=1 \mathrm{bpcu}$ ( 4 bits are transmitted in 4 time slots).

The proposed suboptimum non-coherent MU detector was compared with its ML counterpart and both schemes were also compared with a non-coherent user time division multiplexing (TDM) scheme. To do this, we obtained 
the effective transmission rates of both users, defined from the block-error-rate (BLER) as $E T R=R_{\mathrm{c}}(1-B L E R)$. The ETR regions of the three evaluated schemes are depicted in Figure 1 for a fixed SNR value for both users equal to $\rho_{k}=10 \mathrm{~dB}, k=1,2$. For the first two schemes we set $0 \leq \gamma_{1} \leq 1$ and $\gamma_{2}=1-\gamma_{1}$ to obtain the desired range of BLER values. It can be observed that the two setups carrying out non-coherent MU detection strongly outperform the TDM scheme in terms of effective sum-rate, reinforcing the interest of MU non-coherent transmission. Note that the maximum transmission rate of $1 \mathrm{bpcu}$ is never reached in Figure 1 since the minimum BLER of SU detection is never zero at $\rho_{k}=10 \mathrm{~dB}$. Other $\rho_{k}$ values will lead to different maximum effective user rates for each independent user.

Next, we studied the additional MU gain attained in the downlink non-coherent setup. To this end, the BLER performance of user 1 was evaluated for a set of detectors, fixing the transmitted power of user 1 to $0 \mathrm{~dB}$ and varying the transmitted power of user 2 within a range of values. In Figure 2, the BLER values with both the proposed MU non-coherent receiver and the ML MU detector were compared with the SU case in which only non-coherent transmission to user 1 happens. It can be observed that non-coherent SU transmission is the bestperformance achieving scheme for user 2 transmitted power values lower than $5 \mathrm{~dB}$. For transmitted power values of user 2 higher than this value, the ML MU non-coherent detector has better BLER performance, meaning that user 1 can benefit from the transmission to user 2 to enhance its own data detection. The proposed MU detector also exhibits some performance gain over the SU case at transmitted power values of user 2 higher than $18 \mathrm{~dB}$. It can be also observed that the proposed reduced-complexity MU detector approaches the performance of ML detection, especially as the difference between the power sharing factors of both users increases. We highlight here that the latter results support the main conclusion drawn from Section IV regarding the additional MU gain attained in downlink non-coherent systems. Recall that this is only part of the overall gain shown in Figure 1, which already showed the advantage of MU non-coherent communication against TDM.

\section{CONCLUSION}

This letter proposed the use of superposition coding and a detection scheme to enable MU downlink communication in a non-coherent MIMO system. The ML detection rule was also derived, exhibiting exponential complexity with the number of users. In contrast, the complexity of the proposed suboptimum non-coherent MU detector is linear, involving a substantial complexity reduction. The presented MU non-coherent MIMO schemes were shown to increase the overall performance with respect to TDM. Furthermore, downlink MU non-coherent detection is able to provide, in some cases, some gain in the individual user rates with respect to SU detection, thanks to channel knowledge obtained from transmissions to other users.

Overall, this work showed that MU non-coherent detection is feasible and advantageous. The proposed approach is one step towards the implementation of MU non-coherent techniques in real-world cellular systems. Future work includes designs for the non-coherent uplink, further complexity reductions in the MU detection stage and also finding the conditions where downlink MU non-coherent detection provides additional gain in the individual user rates with respect to $\mathrm{SU}$ detection. 


\section{REFERENCES}

[1] G. Caire, N. Jindal, M. Kobayashi, and N. Ravindran, "Multiuser MIMO achievable rates with downlink training and channel state feedback," IEEE Trans. Inf. Theory, vol. 56, no. 6, pp. 2845-2866, June 2010.

[2] "MIMO and smart antennas for mobile systems," 4G Americas, White Paper, June 2013.

[3] P. Popovski and E. de Carvalho (Editors), "D3.1: Positioning of multi-node/multi-antenna transmission technologies," ICT-317669-METIS, Project Deliverable, Aug. 2013.

[4] L. Hanzo, M. El-Hajjar, and O. Alamri, "Near-capacity wireless transceivers and cooperative communications in the MIMO era: Evolution of standards, waveform design, and future perspectives," Proceedings of the IEEE, pp. 1343-1385, Aug. 2011.

[5] T. L. Marzetta and B. M. Hochwald, "Capacity of a mobile multiple-antenna communication link in Rayleigh flat fading," IEEE Trans. Inf. Theory, vol. 45, no. 1, p. 139157, Jan. 1999.

[6] B. M. Hochwald and W. Sweldens, "Differential unitary space-time modulation," IEEE Trans. Commun., vol. 48, no. 12, pp. 2041-2052, Dec. 2000.

[7] T. Cui and C. Tellambura, "Bound-intersection detection for multiple-symbol differential unitary space-time modulation," IEEE Trans. Commun., vol. 53, no. 12, pp. 2114-2123, Dec. 2005.

[8] M. Beko, J. Xavier, and V. Barroso, "Non-coherent communication in multiple-antenna systems: Receiver design and codebook construction,” IEEE Trans. Signal Process., vol. 55, no. 12, pp. 5703-5715, Dec. 2007.

[9] I. Kammoun, A. Cipriano, and J. Belfiore, "Non-coherent codes over the Grassmannian," IEEE Trans. Wireless Commun., vol. 6, no. 10, pp. 3657-3667, Oct. 2007.

[10] R. H. Gohary and T. N. Davidson, "Non-coherent MIMO communication: Grassmannian constellations and efficient detection," IEEE Trans. Inf. Theory, vol. 55, no. 3, pp. 1176-1205, Mar. 2009.

[11] T. Cover, "Broadcast channels," IEEE Trans. Inf. Theory, vol. 18, no. 1, pp. 2-14, Jan. 1972.

[12] S. Vanka, S. Srinivasa, Z. Gong, P. Vizi, K. Stamatiou, and M. Haenggi, "Superposition coding strategies: Design and experimental evaluation," IEEE Trans. Wireless Commun., vol. 11, no. 7, pp. 2628-2639, July 2012. 


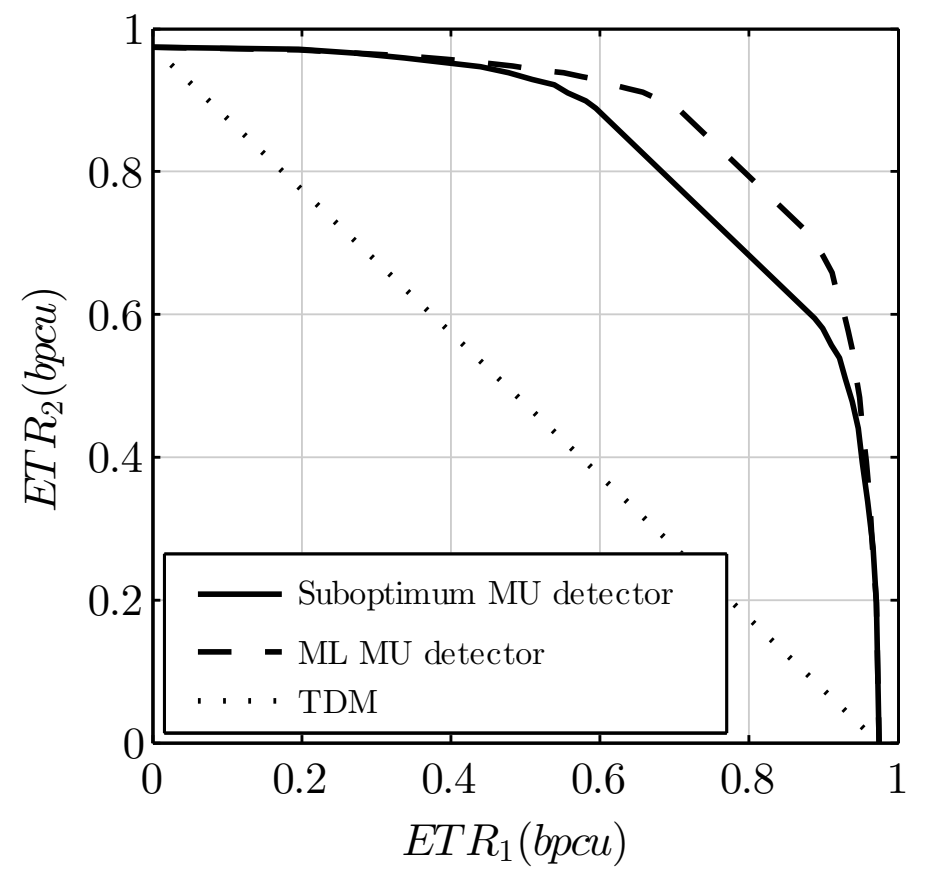

Fig. 1. Effective transmission rates in a two-user non-coherent downlink communication with GC using TDM, superposition coding with ML MU detection and superposition coding with proposed MU detection. 


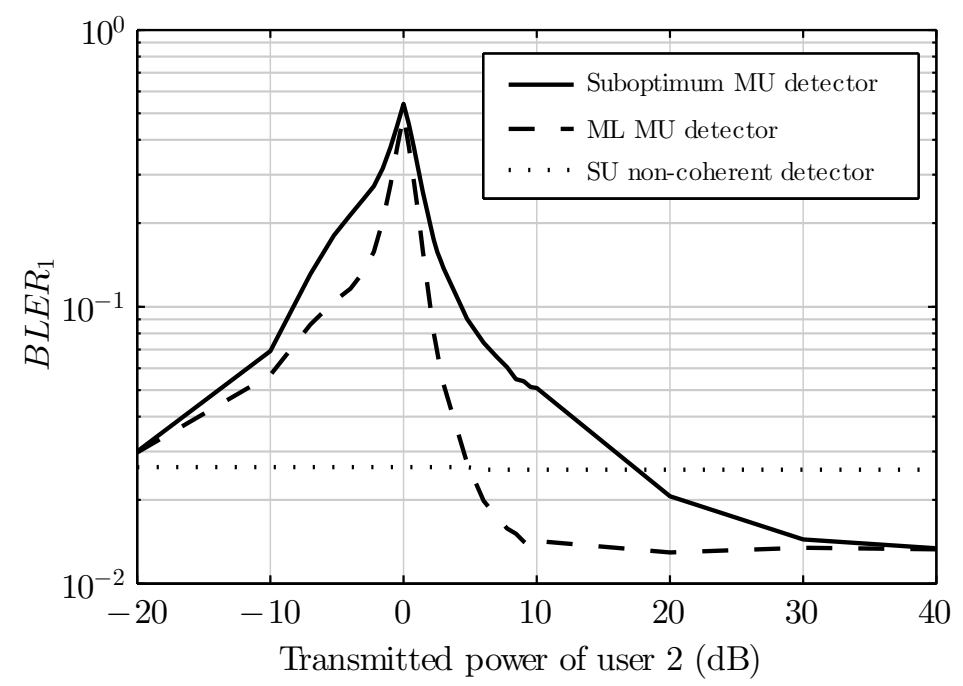

Fig. 2. BLER performance for the detection schemes under study, as a function of the transmitted power of user 2 . 